\title{
Selective excitation and control of coherent terahertz Smith-Purcell radiation by high- intensity period-tunable train of electron micro-bunches
}

Cite as: Appl. Phys. Lett. 113, 171104 (2018); https://doi.org/10.1063/1.5054583

Submitted: 31 August 2018 . Accepted: 07 October 2018 . Published Online: 23 October 2018

Yifan Liang, Yingchao Du (D), Dan Wang, Lixin Yan (D), Qili Tian, Kai Chen, Wenhui Huang (D), Chuanxiang Tang, I. V. Konoplev (D), H. Zhang, and G. Doucas

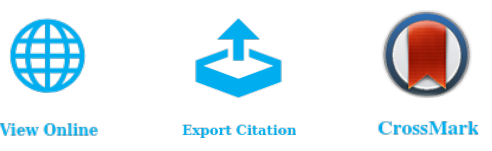

\section{ARTICLES YOU MAY BE INTERESTED IN}

Terahertz-driven ultrafast recovery of plasmon resonance in photoexcited nanoantennas on GaAs

Applied Physics Letters 113, 171103 (2018); https://doi.org/10.1063/1.5048011

Spin polarization modulation for high-speed vertical-cavity surface-emitting lasers Applied Physics Letters 113, 171102 (2018); https://doi.org/10.1063/1.5040914

Frequency-selective excitation of high-wavevector phonons

Applied Physics Letters 113, 171901 (2018); https://doi.org/10.1063/1.5047447

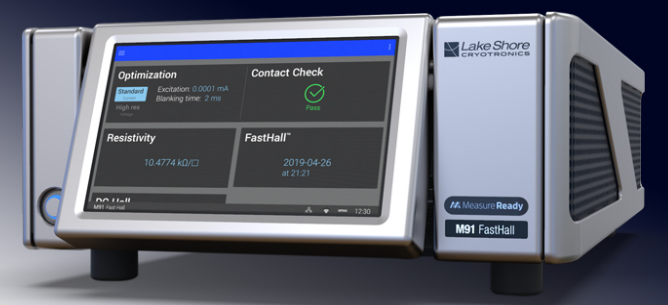

Now Measure Ready M91 FastHall ${ }^{\mathrm{TM}}$ Controller

A revolutionary new instrument for complete Hall analysis

See the video 0 


\title{
Selective excitation and control of coherent terahertz Smith-Purcell radiation by high-intensity period-tunable train of electron micro-bunches
}

\author{
Yifan Liang, ${ }^{1}$ Yingchao Du, ${ }^{1}$ Dan Wang, ${ }^{1}$ Lixin Yan, ${ }^{1, a)}$ Qili Tian, ${ }^{1}$ Kai Chen, ${ }^{1}$ \\ Wenhui Huang, ${ }^{1}$ Chuanxiang Tang, ${ }^{1}$ I. V. Konoplev ${ }^{2, a)}$ H. Zhang, ${ }^{2}$ and G. Doucas ${ }^{2}$ \\ ${ }^{1}$ Department of Engineering Physics, Tsinghua University, Beijing 100084, China \\ ${ }^{2}$ JAI, Department of Physics, University of Oxford, Oxford OX1 3RH, United Kingdom
}

(Received 31 August 2018; accepted 7 October 2018; published online 23 October 2018)

\begin{abstract}
We report the observation and studies of selective excitation and control of terahertz $(\mathrm{THz})$ coherent Smith-Purcell radiation (cSPr) from a train of sub-picosecond-period electron microbunches. The coherence of the radiation from such a train has been demonstrated. The spectrum of cSPr was measured and the selective excitation of the first and the second harmonics was observed, respectively. We also demonstrate experimentally that radiation pulses generated by each electron micro-bunch interfere coherently with the maximum intensity of cSPr observed at the frequency equal to the frequency of the micro-bunch spacing. The experimental results greatly contribute to the understanding of coherent Smith-Purcell radiation from the train of electron micro-bunches, as well as the development of THz diagnostics for accelerators. Published by AIP Publishing.
\end{abstract}

https://doi.org/10.1063/1.5054583

In recent years, there has been substantial interest in generating high power, narrow-band but broadly tunable THz radiation. One of the ways to achieve this is by using trains of well controlled, periodic, electron micro-bunches, i.e., electron beam consisting of equally spaced microbunches. ${ }^{1-11}$ Effective methods of generating intense $\mathrm{THz}$ radiation with such an electron beam include coherent Transition radiation (cTr), Cherenkov radiation, synchrotron radiation, radiation from undulators, and coherent SmithPurcell radiation (cSPr). The coherent transition radiation is a simple and effective method to generate pulses of intense $\mathrm{THz}$ radiation and has been widely used as the basic tool for beam diagnostics. ${ }^{1-6}$ The transition radiation occurs when the electron beam passes through a metal foil, which may cause a number of issues from beam loss and deterioration to foil piercing. Also the width of the spectrum line generated is defined by the number of micro-bunches in the train (normally around 10) limiting its application where the narrow line is required. The attractive solution for many applications is utilizing radiation mechanisms in which radiating structures may not destroy the beam and play a more significant role in determining the radiation characteristics. Although the ideas of utilizing trains of electron micro-bunches to generate coherent radiation in radiating structures were put forward, ${ }^{5,6,10-12}$ a limited number of experiments have been carried out due to the difficulty in obtaining and controlling high-intensity pre-bunched relativistic electron beams.

In this letter, we report the results of first studies of the influence of micro-bunch periodicity on the amplitude of the first and the second harmonics of cSPr measured at a specific point. The choice of SP radiation is attributed to the facts that the SP radiation mechanism allows a number of possibilities in controlling the properties of the generated radiation. ${ }^{2,12-16}$ This mechanism can also be explored to develop

\footnotetext{
${ }^{\text {a) }}$ Authors to whom correspondence should be addressed: yanlx@mail.tsinghua. edu.cn and Ivan.Konoplev@physics.ox.ac.uk
}

a promising femtosecond electron beam diagnostic tool to monitor the longitudinal (time) profile or periodicity of the pre-bunched beam. ${ }^{2,17-20}$ In 2005, the concept of frequencylocked cSPr induced by electron bunch trains was put forward, with the bunch spacing equal to $1.75 \mathrm{~cm}$ (Ref. 21) (one bunch in one accelerating bucket) which was much larger than the radiation wavelength. Under this condition, the pulse repetition rate increases but the pulse peak power remains almost the same as the one generated by a single micro-bunch and no radiation tunability can be achieved. Recent advances in producing a tunable high-intensity train of electron micro-bunches with sub-picosecond spacing (the whole train is in one accelerating bucket) around the world and at the Tsinghua Thomson scattering x-ray source $(\mathrm{TTX})^{5-9,22}$ provide the capability of generating highintensity and narrow-band $\mathrm{THz}$ radiation. Here, a tunable $\mathrm{THz}$ cSPr has been studied both theoretically and experimentally continuing last two decades effort of different research teams to design a high-power tunable $\mathrm{THz}$ source of coherent radiation. We report on the observation of different harmonics of cSPr driven by seven equally spaced (with subpicosecond distances) electron micro-bunches and demonstrate experimentally both the selective excitation of the first two harmonics of cSPr and the coherent interference of the pulses generated by each of the micro-bunches (good agreement between theory and experiment is demonstrated).

SP radiation is emitted when a charged particle passes in the vicinity of the surface of a metallic grating. The dispersion which defines the relation between relative beam velocity $\beta$, measured wavelength $\lambda$, observation angle $\theta$, and period of the grating $d$ is

$$
\lambda=\frac{d}{m}\left(\beta^{-1}-\cos \theta\right),
$$

where $m$ is the harmonic number of the radiation. The energy $d I$ radiated by a single electron in the solid angle $d \Omega$ can be written as ${ }^{22,24}$ 


$$
\left(\frac{d I}{d \Omega}\right)_{1}=2 \pi q^{2} \frac{Z}{d^{2}} \frac{m^{2} \beta^{3}}{(1-\beta \cos \theta)^{3}} R^{2} \exp \left(-\frac{2 x_{0}}{\lambda_{e}}\right)
$$

where $q$ represents the charge, $\mathrm{Z}$ is the length of the grating, $x_{0}$ is the distance between the electron and the surface of the grating, $R_{n}$ represents the radiation factor or grating "efficiency" of the radiation generation which is the function of the grating geometry and the harmonic number, and $\lambda_{e}$ is the evanescent wavelength (or the beam grating coupling parameter) defined by

$$
\lambda_{e}=\frac{\lambda}{2 \pi} \frac{\beta \gamma}{\sqrt{1+\beta^{2} \gamma^{2} \sin ^{2} \theta \sin ^{2} \varphi}},
$$

where $\gamma=\left(1-\beta^{2}\right)^{-1 / 2}$, and $\varphi$ is the azimuthal angle of emission. In case of a single bunch, the energy is also proportional to the square of the electron number and the bunch form factor. Here in this paper, we consider a picosecond long train consisting of femtosecond long micro-bunches. In this case, the energy radiated will be proportional to the form factor of the train rather than a single micro-bunch.

Considering a train of $N_{b}$ electron bunches and $N_{\mathrm{e}}$ electrons contained in each bunch, the energy generated is

$$
\begin{aligned}
\left(\frac{d I}{d \Omega}\right)_{N_{b}} & =\left(\frac{d I}{d \Omega}\right)_{1}\left(N_{e} S_{i n c}+N_{e}^{2} S_{c o h}\right)\left|\sum_{n=1}^{N_{b}} \exp \left(\frac{i 2 \pi n \lambda_{\mathrm{b}}}{\lambda}\right)\right|^{2} \\
& =\left(\frac{d I}{d \Omega}\right)_{1}\left(N_{e} S_{i n c}+N_{e}^{2} S_{c o h}\right)\left[\frac{\sin \left(\frac{\pi N_{b} \lambda_{\mathrm{b}}}{\lambda}\right)}{\sin \left(\frac{\pi \lambda_{\mathrm{b}}}{\lambda}\right)}\right]^{2}
\end{aligned}
$$

where $S_{i n c}$ is the incoherent factor, $S_{c o h}$ is the coherent factor, and $\lambda_{\mathrm{b}}$ is the distance between adjacent bunches. The form factor of the bunch train can be defined as

$$
F(\lambda)=S_{c o h}(\lambda) \cdot\left[\frac{\sin \left(\frac{\pi N_{b} \lambda_{\mathrm{b}}}{\lambda}\right)}{N_{\mathrm{b}} \sin \left(\frac{\pi \lambda_{\mathrm{b}}}{\lambda}\right)}\right]^{2} .
$$

According to Eq. (4), cSPr intensity is determined by the product of the single particle emission $\left(\frac{d I}{d \Omega}\right)_{1}$, the square of the electron number, and the bunch train form factor $F(\lambda)$. Figure 1 illustrates the expected amplitudes of the first 3 harmonics of the generated cSPr radiation (not electron beam current) at a specific observation point from a train of microbunches (Fig. 1). The insets in Fig. 1 show the schematics of the micro-bunch train with periodicity indicated. It is clear from the comparison of the top and the bottom plot in Fig. 1 that by manipulating the parameters of the train, the amplitudes of the radiation harmonics can be controlled. In this letter, we show that by changing the micro-bunch periodicity, the second harmonic of the cSPr $(m=2)$ but the fundamental harmonic of the electron micro-bunch train is predominantly generated.

The experiments were performed at TTX, ${ }^{7}$ where tunable high-intensity trains consisting of 7 and up-to 15 electron
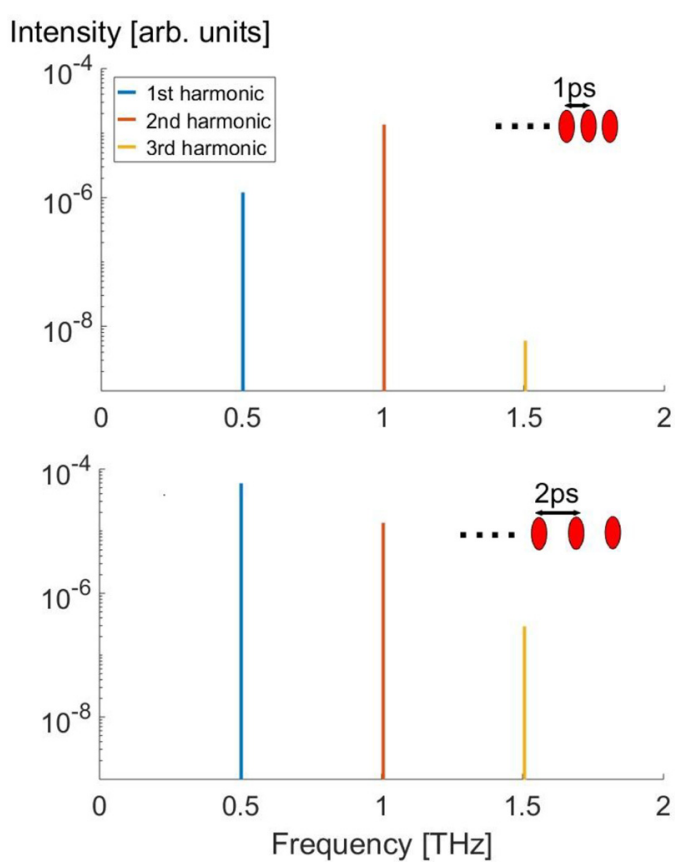

FIG. 1. First three harmonics of cSPr at $90^{\circ}$ observation angle from the 0.6mm-period grating excited by a bunch train with micro-bunch periodicities of $2 \mathrm{ps}$ (bottom) and $1 \mathrm{ps}$ (top), respectively. The longitudinal distribution of micro-bunches is supposed to be Gaussian and the rms length is $0.3 \mathrm{ps}$.

micro-bunches can be produced based on nonlinear longitudinal space charge oscillations. ${ }^{7,8,10}$ This method utilizes nonlinear plasma oscillation experienced by the beam that has a strong initial longitudinal charge density modulation induced at the cathode by an amplitude modulated laser pulse. High peak current spikes are formed after a 1/2 plasma oscillation period and the longitudinal structure is then fixed by quick acceleration of beam energy to $45 \mathrm{MeV}$. The total beam charge can be varied from tens of $\mathrm{pC}$ to $1 \mathrm{nC}$. The periodic spacing between the micro-bunches in the train can be tuned continuously either by changing the launching phase of the radio-frequency gun or by varying the compression factor of the magnetic chicane. ${ }^{7}$ In our case to vary the cSPr intensity generated by the beam, the micro-bunch spacing was tuned from 0.88 ps to $1.16 \mathrm{ps}$ by varying the launching phase of the rf gun. The measurements described here were performed with the train of 7 electron bunches with a total charge of around $300 \mathrm{pC}$ and density spikes of around $100 \mathrm{~A}$ (peak current). The transverse size of the beam (diameter) at the interaction point, i.e., while propagating above the grating, was around $400 \mu \mathrm{m}$. To maximize the signal intensity for this experimental set-up, i.e., to obtain a strong signal at $90^{\circ}$, the grating blazed angle was chosen to be $45^{\circ} .^{23}$

The dispersion relation Eq. (1) determines the radiation frequency measured at a certain observation angle. If the observation angle is fixed, the measured fundamental frequency $(m=1)$ of the radiation should conserve for a specific grating. It is also clear from Eq. (1) that all high harmonics of this fundamental frequency $(m>1)$ will be also measured (if present) at the same position of the detector. ${ }^{13}$ The cSPr experiment setup is shown in Fig. 2. The vacuum chamber houses the grating, a planar mirror, an off-axis parabolic mirror, and a TPX window. As the trains passed over the metallic grating, the radiation emitted at $90^{\circ}$ 


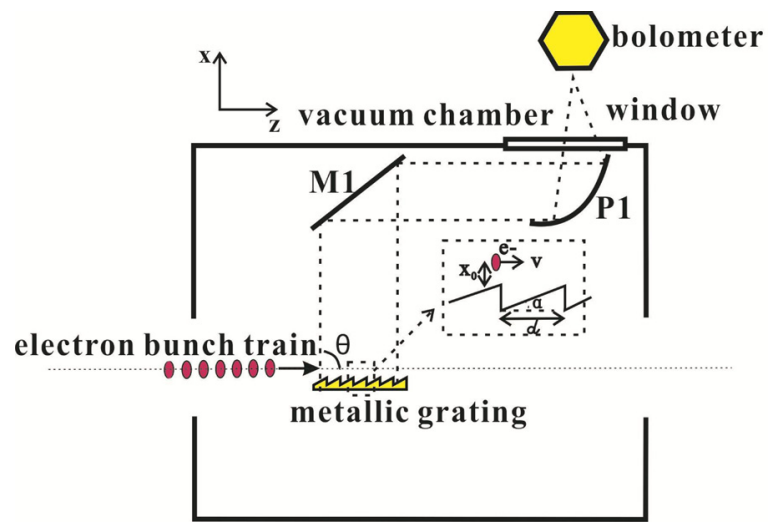

FIG. 2. The schematic of the cSPr experiment setup. Electron bunch trains travel along the $\mathrm{z}$ direction close to a metallic grating. The cSPr emitted at $90^{\circ}$ (observation angle) will be reflected by a planar mirror M1 to an offaxis parabolic mirror $\mathrm{P} 1$ to be focused on the bolometer.

(relative to the beam line) was reflected by the planar mirror and directed to the off-axis parabolic mirror and then it was focused and detected by a bolometer (Fig. 2). The grating can be moved close to or away from the beam by a remotely controlled stepping motor. The observation angle was fixed at $90^{\circ}$ and thus the measured radiation wavelength would be: $\lambda=d / m$. At the initial stage, the spectrum of the cSPr was measured using a Michelson interferometer which was mounted before the bolometer. A typical interferogram and spectrum measured are shown in Fig. 3(a). In the experiment, two gratings of $0.3 \mathrm{~mm}$ and $0.6 \mathrm{~mm}$ period were used. The fundamental harmonic of the former, therefore, coincides with the second harmonic of the latter at $1 \mathrm{THz}$. To observe high radiation intensity and thus to improve the reliability of the measurements, beam parameters as well as the distance between the beam and the gratings were optimized. The dependence of cSPr intensity on the beam-grating separation were measured and shown in Fig. 3(b), observed from a 0.6mm-period grating and driven by the electron bunch train with 1 ps micro-bunch spacing. To get as large signal as possible while still driving the beam with minimal losses, the beam-grating separation $\left(x_{0}\right)$ has been fixed to $0.5 \mathrm{~mm}$. The dependence of cSPr intensity on the total charge of the bunch train was also measured. The quadratic fit demonstrates that the radiation is coherent as described by Eq. (4), i.e., the radiation intensity is proportional to $N_{e}^{2} N_{\mathrm{b}}^{2}$.

By tuning the bunch train spacing, the amplitude variation of the cSPr was observed, which is the result of the interference of the pulses generated by each micro-bunches. In Figs. 4(a) and 4(b), the comparison of theoretical predictions (blue solid line), based on the analysis of the bunch train form factor (simulation by $\mathrm{ASTRA}^{25}$ ) at $1 \mathrm{THz}$, and experimental measurements of the first [Fig. 4(a)] and the second [Fig. 4(b)] harmonics are demonstrated. By changing the distance between the micro-bunches, the variation of the amplitudes of the harmonics has been observed with the maximum signal in both cases measured at $1 \mathrm{ps}$ micro-bunch periodicity, coinciding with the expected frequency at this observation angle for the both gratings. A good agreement between the measured data [dots in Figs. 4(a) and 4(b)] and the simulated form factor (solid lines) can be seen. The small deviations can be attributed to the accuracy of the formation

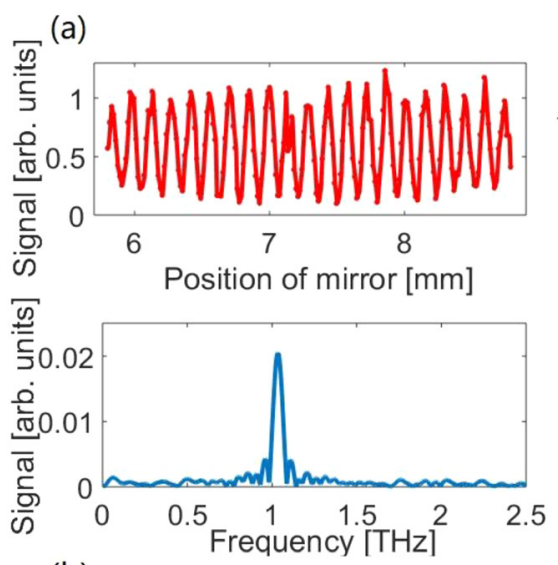

(b)

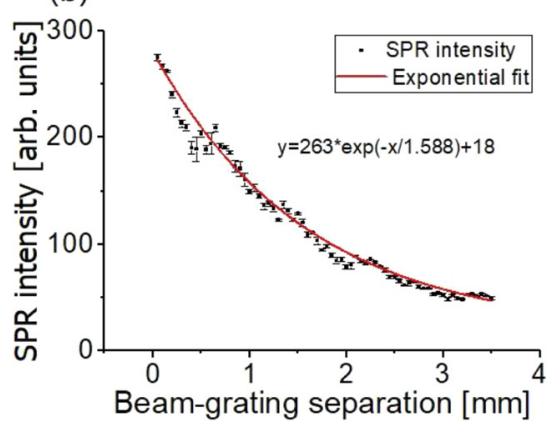

(c)

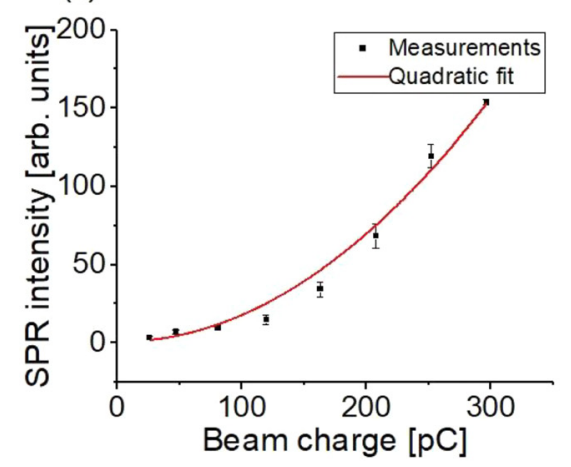

FIG. 3. (a) Autocorrelation function (top) and the THz spectrum (bottom) of cSPr measured with a $0.3-\mathrm{mm}$-period grating and 1-ps-spacing bunch trains. Typical dependences of the intensity of cSPr (with $1 \mathrm{THz}$ band-pass filter) on (b) the beam-grating separation and (c) the total beam charge. The cSPr measured in (b) and (c) was generated by a 1-ps-spacing electron bunch train propagating in the vicinity of the $0.6-\mathrm{mm}$-period grating.

of an individual micro-bunch in the train and/or positioning of the micro-bunches. In both cases, to improve reproducibility of the experiments, the same set-up has been used to measure the radiation intensity at $1 \mathrm{THz}$. The measurements were carried out using a broadband bolometer that has uniform response in the frequency range from $0.1 \mathrm{THz}$ up to $3 \mathrm{THz}$. The results shown in Fig. 4(a) were observed from the $0.3-\mathrm{mm}$-period grating emitting $1 \mathrm{THz}$ radiation (the first harmonic) at $90^{\circ}$ observation angle. The measured interferogram of the cSPr signal from a 0.3-mm-period grating and 1ps-spacing bunch trains is shown in Fig. 3(a), illustrating a conventional case in which most of the pulse energy was concentrated in the first harmonic of the grating $(m=1)$. According to the dispersion relation Eq. (1), if the 0.3-mmperiod grating is substituted with the $0.6-\mathrm{mm}$-period grating, the first harmonic of $\mathrm{cSPr}(m=1)$ will be at $f=0.5 \mathrm{THz}$ while the second harmonic $(m=2)$ will be at $f=1 \mathrm{THz}$. The 

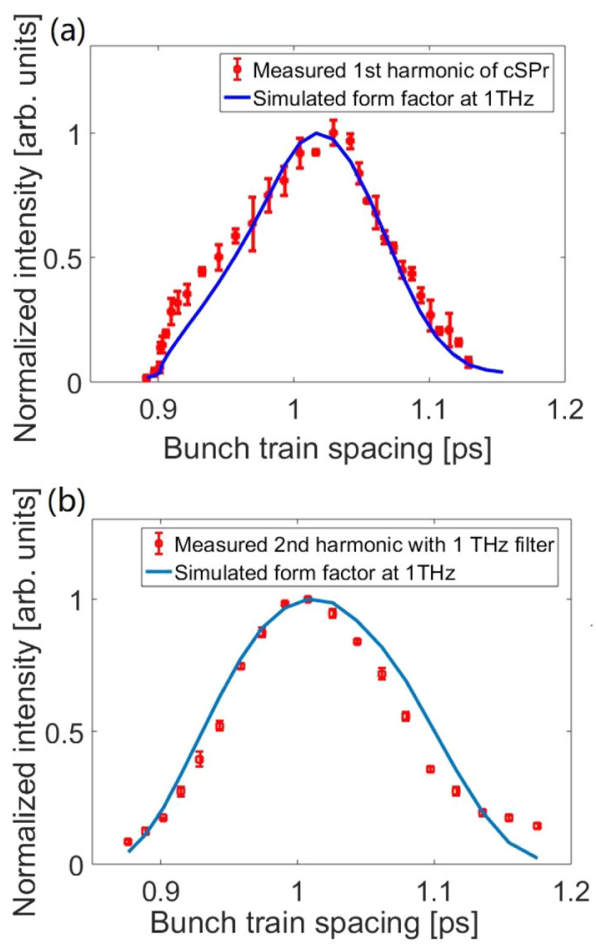

(c)

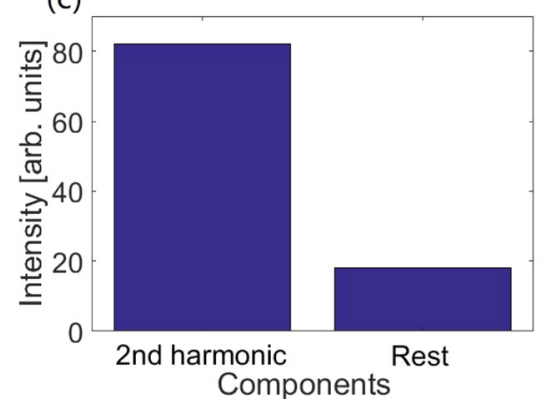

FIG. 4. (a) Dependence of cSPr energy (the fundamental harmonic) on the 0.3 -mm-period grating, measured at $90^{\circ}$ observation angle, on the spacing between micro-bunches in the train. The theoretical curve (solid line) is the bunch train form factor from ASTRA simulation. (b) Dependence of the energy of the second harmonic of cSPr from the 0.6-mm-period grating on the spacing between the micro-bunches in the train with a $1 \mathrm{THz}$ narrowband-pass filter used in front of the bolometer. The theoretical curve (solid line) is the bunch train form factor from the ASTRA simulation. (c) The split of the energy contents in the pulse between the second harmonic and the rest components from the $0.6-\mathrm{mm}$-period grating and the 1-ps-spacing bunch train.

ratios of energy distribution between frequency components were measured and it was found that in the case of $0.6-\mathrm{mm}$ period grating, the second harmonic had significantly larger [Fig. 4(c)] energy contents as compared with the rest of the components. This is in a brilliant agreement with theoretical predictions in which both harmonics should be excited [Fig. 1(b)] with the second harmonic $(1 \mathrm{THz})$ having higher amplitude as compared with the first harmonic $(0.5 \mathrm{THz})$. The experimental results of the excitation of the second harmonic $(m=2)$ of 0.6 -mm-period grating by the 1-ps-spacing microbunch train, show that the signal amplitude measured at $1 \mathrm{THz}$ was $300 \mathrm{mV}$ while the amplitude of the total signal (1 THz filter removed) was $470 \mathrm{mV}$. Using the filter specification $^{26}$ and the transmission efficiency at this frequency $(\sim 78 \%)$ gave us an estimation of around 300/0.78/470 $\approx 82 \%$ of the total energy being in the second harmonic of cSPr
[Fig. 4(c)]. The rest of the components would come from the other harmonics of cSPr and the transition radiation which occurred when the electron beam passed by the grating.

In summary, we have presented the first observation of selective excitation of the first two harmonics of cSPr by utilizing tunable electron bunch trains. We demonstrate the coherent radiation from the continuous train of electron micro-bunches having overall length much longer as compared with the operating wavelength and demonstrated the capability in controlling the amplitudes of cSPr harmonics. The beam parameters, such as the total beam charge, were varied and no coherence loss of the generated radiation was observed. To show this, the quadratic increase in the coherent radiation intensity with the increase in the beam total charge was experimentally verified. Using both theoretical predictions and experimental data, it was demonstrated that the second harmonic of cSPr can be selectively excited if the micro-bunch periodicity coincides with the radiation harmonic wavelength. In this case, the grating operates as an oscillator and dispersive media allowing excitation of the certain harmonic of cSPr determined by the micro-bunch periodicity. In principle, the results can be extrapolated to the selective excitation of even higher harmonics of cSPr, and may be not restricted to the blazed grating but also applied to other types of gratings and two dimensional metasurfaces, ${ }^{16,27,28}$ allowing design of tunable $\mathrm{THz}$ radiation sources. These experimental results provide further understandings of cSPr and will significantly promote the advances in generating a compact, high-intensity, and tunable light source driven by electron bunch trains.

This work was supported by the National Natural Science Foundation of China (NSFC Grant Nos. 11835004, 11475097, 11375097, and 11435015) and the National Key Scientific Instrument and Equipment Development Project of China (Grant No. 2013YQ12034504). I. V. Konoplev would like to acknowledge the partial support of the project from the STFC UK through the JAI Grant (No. ST/J002011/1) and the Leverhulme Trust through the International Network Grant No. IN-2015-012. H. Zhang would like to thank NUDT (China) for supporting his DPhil project.

${ }^{1}$ P. Muggli, V. Yakimenko, M. Babzien, E. K. Kallos, and K. P. Kusche, Phys. Rev. Lett. 101, 054801 (2008).

${ }^{2}$ H. Zhang, I. V. Konoplev, A. J. Lancaster, H. Harrison, G. Doucas, A. Aryshev, M. Shevelev, N. Terunuma, and J. Urakawa, Appl. Phys. Lett. 111, 043505 (2017); Y. Liang, Y. Du, X. Su, D. Wang, L. Yan, Q. Tian, Z. Zhou, D. Wang, W. Huang, W. Gai, C. Tang, I. V. Konoplev, H. Zhang, and G. Doucas, Appl. Phys. Lett. 112, 053501 (2018).

${ }^{3}$ Y. E. Sun, P. Piot, A. Johnson, A. H. Lumpkin, T. J. Maxwell, J. Ruan, and R. Thurman-Keup, Phys. Rev. Lett. 105, 234801 (2010).

${ }^{4}$ Y. Shen, X. Yang, G. L. Carr, Y. Hidaka, J. B. Murphy, and X. Wang, Phys. Rev. Lett. 107, 204801 (2011).

${ }^{5}$ P. Piot, Y. E. Sun, T. J. Maxwell, J. Ruan, A. H. Lumpkin, M. M. Rihaoui, and R. Thurman-Keup, Appl. Phys. Lett. 98, 261501 (2011).

${ }^{6}$ S. Antipov, M. Babzien, C. Jing, M. Fedurin, W. Gai, A. Kanareykin, K. Kusche, V. Yakimenko, and A. Zholents, Phys. Rev. Lett. 111, 134802 (2013).

${ }^{7}$ Z. Zhang, L. Yan, Y. Du, Z. Zhou, X. Su, L. Zheng, D. Wang, Q. Tian, W. Wang, J. Shi et al., Phys. Rev. Lett. 116, 184801 (2016).

${ }^{8}$ P. Musumeci, R. K. Li, and A. Marinelli, Phys. Rev. Lett. 106, 184801 (2011). 
${ }^{9}$ M. Dunning, C. Hast, E. Hemsing, K. Jobe, D. McCormick, J. Nelson, T. O. Raubenheimer, K. Soong, Z. Szalata, D. Walz et al., Phys. Rev. Lett. 109, 074801 (2012).

${ }^{10}$ P. Musumeci, R. K. Li, K. G. Roberts, and E. Chiadroni, Phys. Rev. Spec. Top. Accel. Beams 16, 100701 (2013).

${ }^{11}$ G. Andonian, O. Williams, X. Wei, P. Niknejadi, E. Hemsing, J. B. Rosenzweig, P. Muggli, M. Babzien, M. Fedurin, K. Kusche et al., Appl. Phys. Lett. 98, 202901 (2011).

${ }^{12}$ H. Zhang, I. V. Konoplev, G. Doucas, and J. Smith, Phys. Plasmas 25, 043111 (2018).

${ }^{13}$ W. Li, Y. Xu, Y. Lu, Z. He, Q. Jia, and L. Wang, J. Infrared, Millimeter, Terahertz Waves 38, 12 (2017).

${ }^{14}$ Y. Li, Y.-E. Sun, and K.-J. Kim, Phys. Rev. Spec. Top. Accel. Beams 11, 080701 (2008).

${ }^{15}$ A. Aryshev, A. Potylitsyn, G. Naumenko, M. Shevelev, K. Lekomtsev, L. Sukhikh, P. Karataev, Y. Honda, N. Terunuma, and J. Urakawa, Phys. Rev. Accel. Beams 20, 024701 (2017).

${ }^{16}$ Z. Wang, K. Yao, M. Chen, H. Chen, and Y. Liu, Phys. Rev. Lett. 117, 157401 (2016).

${ }^{17}$ G. Doucas, V. Blackmore, B. Ottewell, C. Perry, P. G. Huggard, E. Castro-Camus, M. B. Johnston, J. L. Hughes, M. F. Kimmitt, B. Redlich et al., Phys. Rev. Spec. Top. Accel. Beams 9, 092801 (2006).
${ }^{18}$ H. L. Andrews, F. Bakkali Taheri, J. Barros, R. Bartolini, V. Bharadwaj, C. Clarke, N. Delerue, G. Doucas, N. Fuster-Martinez, M. VieilleGrosjean et al., Phys. Rev. Spec. Top. Accel. Beams 17, 052802 (2014).

${ }^{19}$ S. E. Korbly, A. S. Kesar, R. J. Temkin, and J. H. Brownell, Phys. Rev. Spec. Top. Accel. Beams 9, 022802 (2006).

${ }^{20}$ G. Doucas, J. H. Mulvey, M. Omori, J. Walsh, and M. F. Kimmitt, Phys. Rev. Lett. 69, 1761 (1992).

${ }^{21}$ S. E. Korbly, A. S. Kesar, J. R. Sirigiri, and R. J. Temkin, Phys. Rev. Lett. 94, 054803 (2005).

${ }^{22}$ L. Zsolt, A. Andreev, I. V. Konoplev, A. Seryi, and J. Smith, Plasma Phys. Controlled Fusion 60, 075012 (2018).

${ }^{23}$ J. H. Brownell and G. Doucas, Phys. Rev. Spec. Top. Accel. Beams 8, 091301 (2005).

${ }^{24}$ J. H. Brownell, J. Walsh, and G. Doucas, Phys. Rev. E 57, 1075 (1998).

${ }^{25}$ See http://www.desy.de/ mpyflo for description of software package ASTRA and manual for the software package ASTRA.

${ }^{26}$ See https://www.thorlabschina.cn/newgrouppage9.cfm?objectgroup_id=7611 for description and calibration of filters.

${ }^{27}$ I. V. Konoplev, L. Fisher, A. W. Cross, A. D. R. Phelps, K. Ronald, and C. W. Robertson, Appl. Phys. Lett. 96, 261101 (2010).

${ }^{28}$ I. V. Konoplev, A. J. MacLachlan, C. W. Robertson, A. W. Cross, and A. D. R. Phelps, Phys. Rev. A 84, 013826 (2011). 